July 2009

\title{
Management of Hypothalamic/Chiasmatic Astrocytomas in Children
}

Peter Amenta MD

Follow this and additional works at: https://jdc.jefferson.edu/jhnj Let us know how access to this document benefits you

\section{Recommended Citation}

Amenta, Peter MD (2009) "Management of Hypothalamic/Chiasmatic Astrocytomas in Children," JHN Journal: Vol. 4 : Iss. 3 , Article 3.

DOI: https://doi.org/10.29046/JHNJ.004.3.003

Available at: https://jdc.jefferson.edu/jhnj/vol4/iss3/3

This Article is brought to you for free and open access by the Jefferson Digital Commons. The Jefferson Digital Commons is a service of Thomas Jefferson University's Center for Teaching and Learning (CTL). The Commons is a showcase for Jefferson books and journals, peer-reviewed scholarly publications, unique historical collections from the University archives, and teaching tools. The Jefferson Digital Commons allows researchers and interested readers anywhere in the world to learn about and keep up to date with Jefferson scholarship. This article has been accepted for inclusion in JHN Journal by an authorized administrator of the Jefferson Digital Commons. For more information, please contact: JeffersonDigitalCommons@jefferson.edu. 


\section{Management of Hypothalamic/ Chiasmatic Astrocytomas in Children}

\author{
Peter S. Amenta, MD \\ Department of Neurological Surgery, Thomas Jefferson University Hospital, Philadelphia, PA
}

The management of pediatric hypothalamic/chiasmatic gliomas is currently a controversial issue within the fields of pediatric neurosurgery, neuro-oncology, and radiation oncology. These discrepancies have provided the fuel for a retrospective study I am currently performing in an attempt to link treatment modalities and long-term outcome.

The common thread driving the inconsistencies in care is that the natural history of pediatric hypothalamic/chiasmatic gliomas is erratic and difficult to predict. The literature shows a wide range of outcomes ranging from rapid progression of disease to indolence over years and in some instances spontaneous regression. A 1995 paper describes the standard approach to treatment employed from 1975 to 1991: biopsy for histologic diagnosis; if low-grade, then limited resection followed by chemotherapy for young children and radiation in older children. Recurrences were treated with either chemotherapy or radiation depending on which modality had not been used previously. In the event that both modalities failed, then radical resection was undertaken.

Over the past thirty years, advances in imaging and treatment have brought different approaches to the treatment of these tumors. Multiple authors have advocated radical surgical resection as the primary treatment modality. Others have suggested watchful waiting, delaying even chemotherapy and radiation until tumor progression becomes evident. Important variables that are considered in determining treatment, include: age at time of diagnosis, neurologic function on presentation, presence/ absence of hydrocephalus, and the ability of family to adhere to endocrine therapy post-treatment.

The evolution of the imaging modalities of CT and MRI have provided greater detail of the anatomy affected by the tumor, while also assisting in planning a surgical approach. Additionally, multiple chemotherapeutic regimens have been used with varying degrees of success. Radiation therapy has also been used successfully, but not without its own set of complications. All three treatment modalities are limited by a paucity of data pertaining to the long-term outcomes in this patient population.

\section{Steps of Literature Search}

Step \#1: Thomas Jefferson University Library Databases. PubMed literature search utilizing the following headings:

\section{Subject Headings Used:}

1. Chiasmatic glioma AND surgical treatment

2. Hypothalamic glioma AND radiation

3. Hypothalamic glioma AND chemotherapy

4. Chiasmatic glioma AND treatment AND outcomes

Limiting Factors Utilized:

1. Restricted date from 1975 - current

2. Restricted to journals only

Step \#2: Searched the bibliographies of the articles in the database literature search for additional relevant articles. Once all articles were reviewed, I selected the articles that best addressed my question.

\section{Guidelines Selected}

1. Observation without intervention alone may be used from the time of first presentation to the time of first tumor progression. ${ }^{3}$
2. Initial surgical management should be limited to acquisition of a tissue diagnosis and decompression of the optic apparatus and/or the ventricular system..$^{1,2}$

\section{Guideline Review}

1. Observation without intervention alone may be used from the time of first presentation to the time of first tumor progression. ${ }^{3}$

Traditionally, radiation has served as the standard therapy for progressive pediatric hypothalamic/ chiasmatic gliomas. However, there are multiple long-term sequelae of radiation, including: endocrinopathy, vasculopathy, optic nerve injury, and radiation-induced secondary neoplasms. All of these complications must be considered when planning treatment, as the majority of pediatric hypothalamic/chiasmatic glioma patients will survive longer than 10 years. Additionally, most children are diagnosed under the age of five, an age at which they are more likely to experience the long-term sequelae of radiotherapy. As a result, alternate treatment modalities must be explored to avoid the need for radiation at an early age.

Janss et al describes the practice of observation without intervention from the time of first presentation to the time of first tumor progression. In children under the age of five, treatment with vincristine and actinomycin-D is initiated at the first signs/symptoms of tumor progression. Neurosurgeons, neuro-oncologists, and radiation oncologists attempt to use surgery with or without chemotherapy to halt tumor progression before resorting to radiation. Either radical resection or biopsy and limited resection, at the time of presentation, confirms the diagnosis histologically. To limit coginitive deficits secondary to radiotherapy, most children are allowed to age to at least the age of five before starting their course of radiotherapy treatment.

2. Initial surgical management should be limited to acquisition of a tissue diagnosis and decompression of the optic apparatus and/or the ventricular system. ${ }^{1,2}$ 


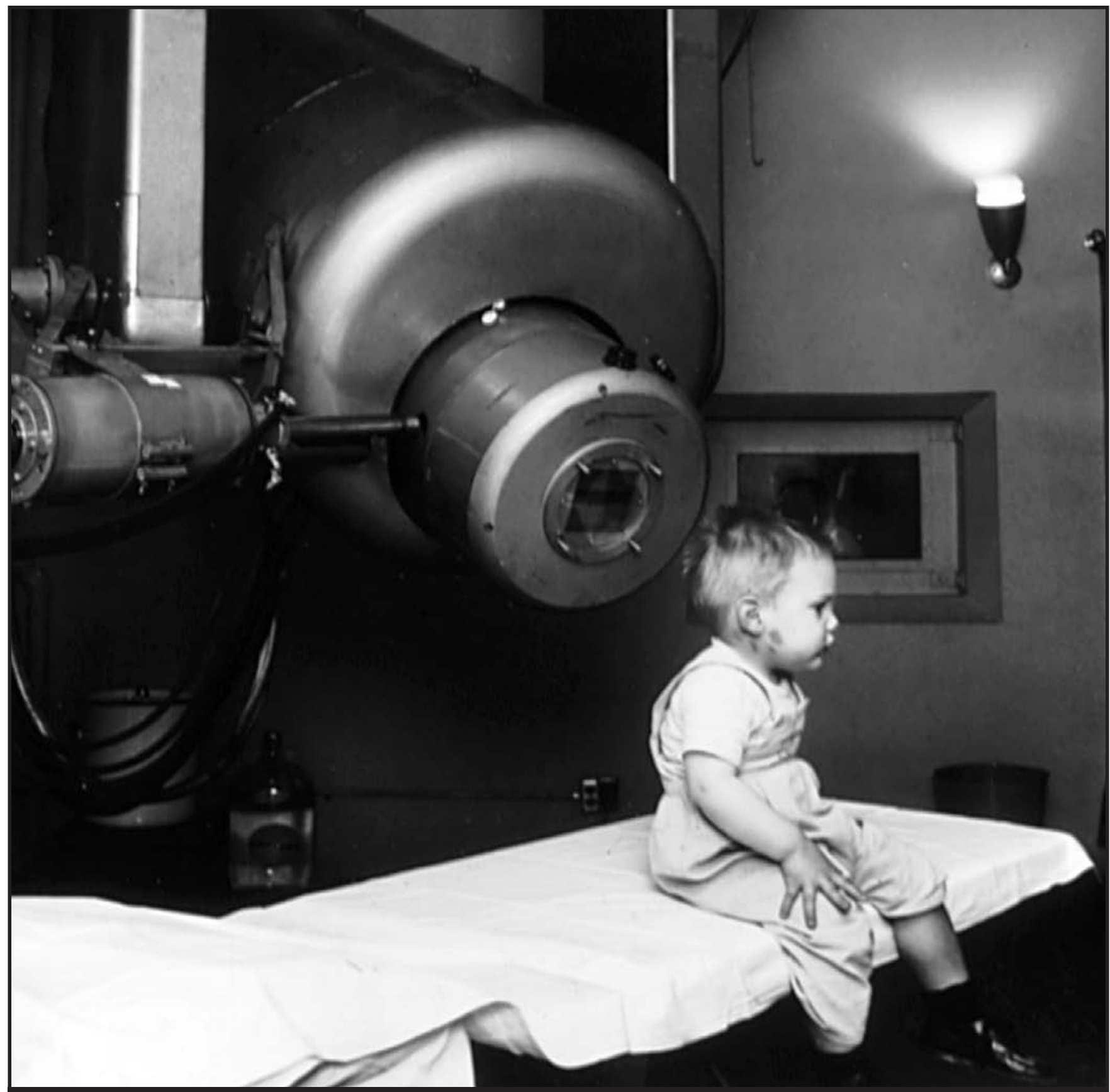

\section{Figure 1}

A pediatric patient and the linear accelerator used to treat his eye tumor (public domain image).

Photo from 1957. Credit: National Cancer Institute

http://visualsonline.cancer.gov/details.cfm?imageid=1924

Multiple centers traditionally advocated surgery for tissue diagnosis and decompression of the optic apparatus and/ or ventricular system. Steinbok et al describes a series of 18 patients with hypothalamic/ chiasmatic gliomas: eight patients had subtotal resections (>95\%), six had partial resections (50-95\%), three had limited resections $(<50 \%)$, and one had no surgery. The authors report an especially high incidence of postoperative complications in those resections of 50\% 
or greater. Patients were found to suffer from DI, SIADH, panhypopituitarism, visual loss, infarction, and fatal cerebral swelling secondary to huge shifts in sodium balance. Debulking procedures $(<50 \%)$ designed at decompressing the optic chiasm showed a trend toward worsening vision. Additionally, there was no difference in the probability of tumor progression or time to progression among the surgical groups undergoing different degrees of resection. The authors argue that there is no evidence to support long-term tumor control with radical resection and thus support a more conservative surgical therapy.

The variability in surgical management discussed throughout the literature is applicable to our institution. At our institution, the surgical approach is practitioner-dependent, with different attendings advocating biopsy/minimal resection or radical surgical resection. We also have the same limitations, as there is no long-term follow-up for patients undergoing radical resection, which has only recently become more popular.

\section{Strengths and Limitations of Primary Research Studies}

\section{Limitation of Both Papers}

The authors of both studies fail to clearly define the condition of the patients prior to and after any intervention. The results of formal testing, such as formal visual fields, endocrine panels, documented presence/ absence of DI or SIADH, and neurocognitive testing are not reported. As a result, the data are difficult to interpret as there is no clear picture of the patients' outcome. Pediatric hypothalamic/ chiasmatic gliomas are difficult to treat because of the variable course the disease follows and the lack of information pertaining to outcomes after surgery, chemotherapy, and radiation. Future studies must include strictly defined criteria to determine the patient's condition at first presentation, morbidity and mortality associated with disease progression, the complications of intervention, and quality of life following those interventions.

\section{Optic pathway and hypothalamic/ chias- matic gliomas in children younger than age five years with a six-year follow-up.}

\section{Strengths}

a. This study offers a comprehensive discussion of findings specific to a small subset of the pediatric population with hypothalamic/chiasmatic gliomas. Through the retrospective analysis, the authors clearly define the issues that are relevant to the treatment of those patients below the age of five.

b. The study draws three important conclusions regarding overall progression of the disease, which still remains the largest unknown in treating this patient population. First, the majority of these patients in this series experienced tumor progression, which suggests a more aggressive course in younger patients. Second, the series shows that chemotherapy can effectively delay the use of radiation. Finally, the authors are able to show that tumors in children with NF-1 are typically less aggressive than tumors in children without NF-1.

\section{Limitations}

a. This series makes no attempt to distinguish between tumors involving the optic chiasm, optic nerves, optic radiations, and hypothalamus. Multiple other studies have shown that the structures involved with these tumors may have an effect on overall prognosis, while also guiding the eventual surgical intervention.

b. This series does not differentiate between invasive and globular/exophytic masses, which is an important distinction when determining extent of surgical resection. Additionally, exophytic tumors may be more amenable to debulking and therefore have an effect on the determination of whether or not to operate.

c. This series does not make a distinction between the different degrees of surgical resection. All patients are grouped together under the heading of "surgery" without identifying patients treated with radical resection versus more conservative measures.

2. Initial surgical management should be limited to acquisition of a tissue diagnosis and decompression of the optic apparatus and/or the ventricular system. ${ }^{1,2}$

\section{Strengths}

a. The authors make an important distinction between tumors based on anatomical location. Patients are divided into two groups: chiasmatic tumors with or without involvement of the optic nerves/ tracts and chiasmatic tumors involving the hypothalamus. This distinction is critical as the authors were able to show differences in clinical presentation, tumor size, and behavior.

b. The authors used a mathematical model to define tumor size pre- and postintervention, thereby clearly defining the following measurements: initial tumor size, percent of tumor resection, percent of tumor progression/regression, and volume of residual tumor.

\section{Limitations}

a. Patients treated with radiation suffered few complications, most likely due to the relatively short follow-up period. Secondary neoplasms and vasculopathy, known complications of radiation therapy in this patient population, were completely absent. The lack of radiationinduced complications portrays better, and possibly inaccurate, outcomes in patients that might show additional sequelae if followed for a longer period of time.

b. The study is limited by the fact that not all patients received the same initial treatment. Some patients received surgery, some chemotherapy, and others observation alone. The authors do not clearly define the criteria driving their treatment modality selection.

\section{References}

1. Sutton LN, Molloy PT, et al. Long-term outcome of hypothalamic/ chiasmatic astrocytomas in children treated with conservative surgery. Journal of Neurosurgery 83: 583-589, 1995.

2. Steinbok $P$, Hentschel S, et al. Management of optic chiasm hypothalamic astrocytomas in children. The Canadie Journal of Neurological Sciences 29: 132-138, 2002.

3. Janss A, Grundy R, et al. Optic pathway and hypothalamic/ chiasmatic gliomas in children younger than age 5 years with a 6-year follow-up. Cancer 75: 1051-1059, 1995.

4. Yasser, K, Hassounah, et al. Optic gliomas: A retrospective analysis of 50 cases. International Journal of Radiation Oncology, Biology, and Physics 56: 807-812, 2003. 